\title{
Defective postsecretory maturation of MUC5B mucin in cystic fibrosis airways
}

\author{
Lubna H. Abdullah, ${ }^{1}$ Jessica R. Evans, ${ }^{2}$ T. Tiffany Wang, ${ }^{1}$ Amina A. Ford, ${ }^{1}$ Alexander M. Makhov, ${ }^{3}$ \\ Kristine Nguyen, ${ }^{1}$ Raymond D. Coakley, ${ }^{1}$ Jack D. Griffith, ${ }^{3}$ C. William Davis, ${ }^{1}$ Stephen T. Ballard, ${ }^{2}$ \\ and Mehmet Kesimer ${ }^{1}$ \\ ${ }^{1}$ Cystic Fibrosis and Pulmonary Diseases Research and Treatment Center, University of North Carolina at Chapel Hill, North \\ Carolina, USA. ²Department of Physiology and Biophysics, College of Medicine, University of South Alabama, Mobile, \\ Alabama, USA. 'ineberger Cancer Center, University of North Carolina at Chapel Hill, North Carolina, USA.
}

In cystic fibrosis (CF), airway mucus becomes thick and viscous, and its clearance from the airways is impaired. The gel-forming mucins undergo an ordered "unpacking/maturation" process after granular release that requires an optimum postsecretory environment, including hydration and $\mathrm{pH}$. We hypothesized that this unpacking process is compromised in the CF lung due to abnormal transepithelial fluid transport that reduces airway surface hydration and alters ionic composition. Using human tracheobronchial epithelial cells derived from non-CF and CF donors and mucus samples from human subjects and domestic pigs, we investigated the process of postsecretory mucin unfolding/maturation, how these processes are defective in CF airways, and the probable mechanism underlying defective unfolding. First, we found that mucins released into a normal lung environment transform from a compact granular form to a linear form. Second, we demonstrated that this maturation process is defective in the CF airway environment. Finally, we demonstrated that independent of $\mathrm{HCO}_{3}{ }^{-}$and $\mathrm{pH}$ levels, airway surface dehydration was the major determinant of this abnormal unfolding process. This defective unfolding/maturation process after granular release suggests that the CF extracellular environment is ion/water depleted and likely contributes to abnormal mucus properties in CF airways prior to infection and inflammation.

Conflict of interest: The authors have declared that no conflict of interest exists.

Submitted: July 26, 2016

Accepted: February 8, 2017

Published: March 23, 2017

Reference information:

JCI Insight. 2017;2(6):e89752. https:// doi.org/10.1172/jici.nsight.89752.

\section{Introduction}

The origin of the thick, sticky airway mucus that characterizes cystic fibrosis (CF) has been the focus of intense study since this disease was first recognized. Much of the CF disease process is thought to be due to a reduction in liquid volume that results from the loss of CF transmembrane conductance regulator (CFTR) function due to mutations in the CFTR gene (1). Recent work, however, indicates that more complicated factors are at play. These factors are secondary to the reduction in volume and are related to changes in the physical properties of the mucus as it becomes concentrated., e.g., alteration of the postsecretory transformation of polymeric mucins into the linear polymers characteristic of healthy, transportable, mucus. This study focuses on the biochemical maturation of mucins, specifically MUC5B, following their granular release using experimental protocols that are physiologically relevant to the native surfaces of healthy and CF airways.

The CFTR protein normally functions as a cAMP-activated $\mathrm{Cl}^{-}$and $\mathrm{HCO}_{3}{ }^{-}$channel that is expressed in the apical membrane of a variety of epithelia, including those of the sweat glands, pancreas, small intestine, and the tracheobronchial airways of the lung. In the airways, CFTR is expressed in the apical membranes of both the ciliated epithelial cells (2) that line the airway surfaces and the serous cells of the airway submucosal glands (3). There are reports of CFTR being expressed in the secretory membranes of airway goblet cells (4), but this possibility has been questioned (5). In CF disease, the loss of CFTR-mediated anion secretion from airway glands and the surface epithelium, combined with ongoing $\mathrm{Na}^{+}$absorption through $\mathrm{ENaC}$ channels in the surface epithelium, is thought to reduce the airway surface liquid (ASL) volume (6).

The polymeric mucins MUC5AC and MUC5B are the major gel-forming macromolecular components of ASL (7). MUC5AC is the product of goblet cells in the surface epithelium $(8,9)$, while MUC5B is a major product of the mucous cells of the submucosal glands (8) and possibly Clara cells in the bronchioles. In primary human tracheobronchial epithelial (HTBE) cell cultures, however, both mucins are products 
of the surface epithelium, and MUC5B is the dominant mucin in the baseline cultures $(5,10)$. These large oligomeric glycoproteins, especially MUC5B, are vital to the lung's defenses (11); they provide an initial layer of innate protection for the underlying epithelium against pathological insults, such as inhaled particles, aerosols, and invading pathogens, which are then removed from the airways via mucociliary transport and cough. In the obstructive lung disease CF, the airway mucus becomes thick (concentrated) and viscous, and its clearance from the airways is impaired (1). The underlying reasons for the compromise of mucus transportability in this disease state have not been fully defined. Our group has previously demonstrated that mucin macromolecules undergo a postsecretory unpackaging process (12) and that mucins are abnormally concentrated in CF lungs (13). The present study was conducted to study in detail the postsecretory processing of these gel-forming mucins, how this process is perturbed in $\mathrm{CF}$, and how qualitative changes in mucin maturation might contribute to decreased airway clearance by cough and ciliary transport in CF.

Oligomeric mucins are packaged tightly into secretory granules in the secretory cells. However, the intergranular organization of these extraordinarily large intracellular molecules, the rapidity of their uncoiling following granular release to form transportable mucus gels, and how this process is affected by postsecretory extracellular conditions are still unclear. Using freshly secreted salivary MUC5B as a model, we previously showed in high-resolution electron micrographs that mucin molecules are stored within secretory granules with their $\mathrm{N}$ - and C-terminal regions arranged in a highly compact granular form and that mucins then unpack and expand rapidly following granular release to form linear molecules (12). Based on these data, we hypothesized that gel-forming mucins undergo an ordered "unpacking" process after granular release that requires an optimal postsecretory environment, including appropriate volume/ hydration and $\mathrm{pH}$ conditions, and that this active process is compromised in the CF lung due to abnormal airway surface hydration and possibly ionic composition.

In the present study, we tested these hypotheses using three approaches. First, we used primary HTBE cells derived from non-CF and CF human donors to test whether mucins undergo a postsecretory unpacking process, as previously proposed (12). Second, we used mucus samples from human CF donors and domestic pigs to test whether this important process is defective in the uninfected CF airway milieu, which is characterized by alterations in the ionic environment and hydration. Third, to better understand the underlying mechanism, we altered the ionic conditions (volume and $\mathrm{pH}$ ) of the epithelial surface by inhibiting transepithelial fluid transport to create a CF-like postsecretory environment in an in vitro system.

\section{Results}

Postsecretory maturation of MUC5B in normal and CF cultures. To determine whether MUC5B mucin undergoes a maturation process after granular release, as previously proposed (12), we used secretions from primary human tracheobronchial cell cultures stimulated by the purinergic agonist ATP $\gamma \mathrm{S}$. After agonist stimulation, the secretions were collected at 2 minutes, 1 hour, and 4 hours. Figure 1A shows the MUC5B distribution in a guanidinium hydrochloride $(\mathrm{GuHCl})$ isokinetic gradient at 2 minutes and at 4 hours. Immediately after secretion ( 2 minutes), approximately $30 \%$ of the MUC5B secreted from non-CF HTBE cell cultures resolved as a compact form at the high-density region at the bottom of the gradient (fractions 10-12), whereas approximately $20 \%$ of the MUC5B resolved as a linear polymeric form at the top (lowdensity) fraction of the gradient. Over time, there was a progressive shift in this distribution, so that at 4 hours, approximately $60 \%$ of the mucin resolved at the top of the gradient as the linearized form (Figure 1A). The distribution of MUC5B in the ATP $\gamma$ S-stimulated CF cultures was similar to that of the 2-minute secretion samples, with MUC5B predominantly found in the high-density "compact" region (Figure 1B). However, in contrast to the MUC5B profile in the 4-hour secretion samples from non-CF cultures, the CF cultures exhibited no progressive shift from the high-density region to the low-density region, suggesting that MUC5B remained predominately in a compact form in the CF cultures (Figure 1B) over that period.

To identify the predominant form of MUC5B in the unstimulated basal secretions that "accumulated" on the non-CF and CF cell surfaces, we analyzed the mucus removed from these cultures prior to experimentation on the same gradient. As shown in Figure 1C, the MUC5B recovered from the non-CF cultures resolved predominately in the low-density region in the linear form, whereas the majority of the MUC5B from the CF HTBE cell cultures was found in the high-density region in the compact form.

Postsecretion unfolding/maturation of MUC5B in non-CF and CF saliva. To validate and compare our findings on MUC5B maturation in airway cell cultures under in vivo conditions, we also evaluated mucin processing in non-CF and CF human saliva, which is an accessible source of MUC5B mucin 


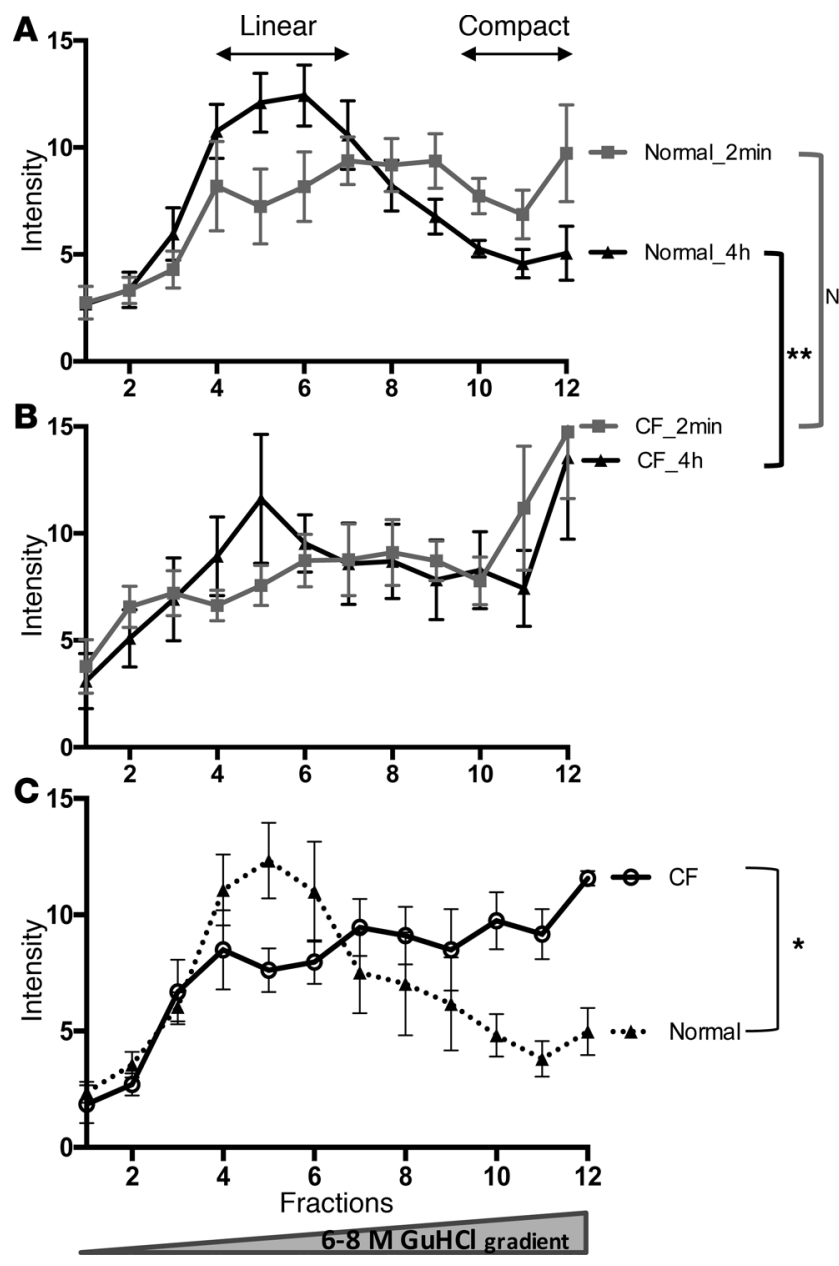

Figure 1. Distribution of the molecular forms of MUC5B following secretion from non-CF and CF HTBE cell cultures. After washing the apical surface, HTBE cells were stimulated apically with $100 \mu \mathrm{M}$ ATP $\gamma S$ to induce mucin secretion. Apical secretions were collected in PBS at 2 minutes and 240 minutes after ATP challenge and immediately added to $4 \mathrm{M} \mathrm{GuHCl}$ to preserve the molecular forms of mucin. Secretions were then subjected to rate zonal centrifugation by a 6-8 $\mathrm{M} \mathrm{GuHCl}$ gradient to separate the molecular forms of MUC5B. (A and B) At 2 minutes, in the ATP $\gamma$ S-stimulated non-CF and CF HTBE cell secretions, MUC5B has similar profiles and is found predominantly in the high-density "compact" region. (B) However, the MUC5B profile in the CF cultures shows no progressive shift from the high-density region to the low-density region, as is observed in the non-CF cultures, suggesting the MUC5B stays predominately in a compact form in the CF cultures $\left({ }^{* *} P\right.$ $=0.01$ ). (C) The distribution of the molecular forms of MUC5B in "accumulated" secretions in non-CF and CF cultures. Unstimulated basal secretions that accumulated on the surface were subjected to the density gradient. The MUC5B profiles indicated that the mucins recovered from the accumulated (72 hours) mucus from non-CF cultures resolved predominately in the low-density region as the linear form, whereas the majority of the MUC5B from CF HTBE cell cultures was found in the same high-density region as the compact form $\left({ }^{*} P=0.01\right)$. SD values are indicated by vertical bars. Unpaired, 2-tailed $t$ test was used to determine statistical significance.

in both populations. Consistent with our previous observations, approximately $70 \%$ of the secreted MUC5B in normal saliva was recovered in linear forms in the low-density fractions of the gradient (fractions 3-6), while 25\% of the MUC5B was recovered in the same middensity region (fractions $7-10$ ) as an intermediate semicompact/semilinear form (Figure 2A). Less than $10 \%$ of normal salivary MUC5B was recovered at the bottom of the gradient, where the compact form is found. In contrast, the MUC5B profile of the saliva from CF patients revealed that approximately $40 \%$ of the MUC5B population was recovered in a linear form, $40 \%$ in the semicompact/semilinear and compact forms, and $20 \%$ at the bottom of the gradient in a compact form (Figure 2A). The distribution of the linear and compact forms was significantly different in the CF saliva versus non-CF saliva $(P<0.01$; Figure $2 \mathrm{~B})$.

Transmission electron microscopy analysis of the mucin conformation reveals a defective MUC5B unpacking process in $C F$. Physical resolution of the distinct MUC5B forms in the pools obtained from rate zonal centrifugation was accomplished using transmission electron microscopy. The compact or semiexpanding granular mucin form of MUC5B was the dominant form found at the bottom of the gradient. In normal saliva, this compact form typically appears as glycosylated mucin subunit chains connected around their $\mathrm{N}$ - and C-terminal protein nodes, forming 10- to 30-nm spherical core structures (Figure 3E). An analysis of the same fractions from the CF samples indicated that the spherical protein nodes/cores were not as well organized and/or processed and were up to 10 times larger $(100-300 \mathrm{~nm}$ ) than normal (Figure 3, C and D). This compact form typically appears as large, irregularly shaped amorphous structures containing an unprocessed protein core. We observed these abnormally compact mucin structures in approximately one-third $(n=4)$ of the CF saliva samples and none of the normal samples.

Effect of the absence of CFTR function on mucin unpacking. To understand the effects of the inhibition of anion secretion on the postsecretory fates of mucins, we used both non-CF HTBE cell cultures and excised domestic pig tracheas. To mimic the defective secretory conditions of the surface epithelium of the $\mathrm{CF}$ airway, HTBE cell cultures were treated with either bumetanide or dimethylamiloride plus acetazolamide $(\mathrm{DMA}+\mathrm{Az})$ to inhibit transepithelial $\mathrm{Cl}^{-}$and $\mathrm{HCO}_{3}{ }^{-}$secretion, respectively. Without inhibitors, the apical secretions exhibited only $10 \%-15 \%$ of the MUC5B in the high-density region. In contrast, the inhibition of $\mathrm{Cl}^{-}$and $\mathrm{HCO}_{3}^{-}$secretion significantly increased the proportion of MUC5B mucin in the high-density compact form region to approximately $30 \%-40 \%(P<0.001$; Figure $4, \mathrm{~A}$ and $\mathrm{B})$. These apical samples also exhibited decreased amounts of the linear MUC5B form found in the low-density region compared with the control $(P<0.01$; Figure $4 \mathrm{~B})$. The combination of bumetanide and DMA+Az treatments increased the 

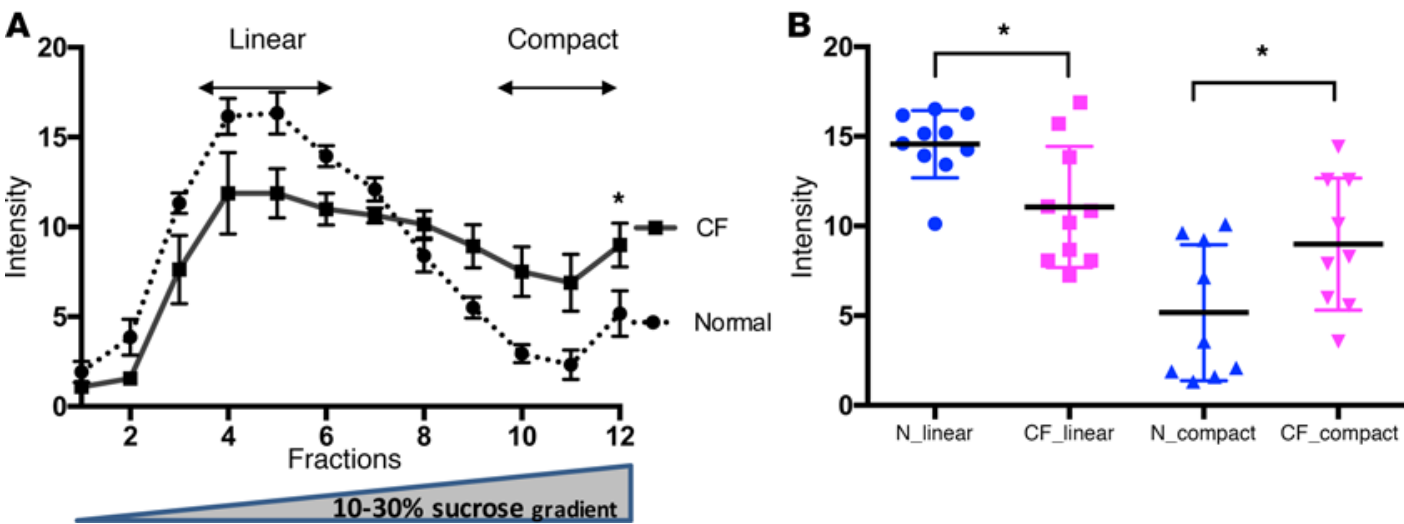

Figure 2. Distribution of the molecular forms of salivary MUC5B from non-CF and CF subjects. (A) Approximately $70 \%$ of the secreted MUC5B population in non-CF saliva was recovered in a linear form in the low-density side of the gradient (fractions 3-6), while $25 \%$ of the MUC5B was recovered in the middensity region (fractions $7-10$ ) in a semicompact/semilinear form. Less than $10 \%$ of the MUC5B was recovered in the bottom of the gradient in a compact form. In contrast, the MUC5B profile of saliva from CF subjects displayed a different profile, with $40 \%$ of the MUC5B population recovered in a linear form, while $40 \%$ of the MUC5B was found in semicompact/semilinear and compact forms. Approximately $20 \%$ of MUC5B from CF saliva was recovered in the bottom region in a compact form ( ${ }^{*} P=0.01$ ). (B) The distribution of the linear and compact forms from normal and CF saliva samples is displayed as a scatter plot ${ }^{*} P=0.012$ ). Mean and standard deviation values are indicated by major and minor horizontal bars respectively. Unpaired, 2 -tailed $t$ test was used to determine statistical significance.

mucus concentration significantly $(P<0.0001)$, from $1.6 \%$ (control) to $3.6 \%$, as measured by percentage solids (Figure 4C). DMA+Az treatment reduced the $\mathrm{pH}$ from 6.8 (control) to 5.6 (Figure 4D; $P<0.0001$ ). No $\mathrm{pH}$ changes were observed after bumetanide treatment.

Porcine tracheas were also used to investigate the effects of transepithelial $\mathrm{Cl}^{-}$and $\mathrm{HCO}_{3}{ }^{-}$secretion on the postsecretion unfolding/maturation of MUC5B. To examine the mechanism underlying Muc5b unpacking and the potential reasons for its defective maturation in the CF extracellular milieu, four inhibitor pretreatment combinations were applied to the basolateral bath: (a) a combination of $100 \mu \mathrm{M}$ bumetanide and $100 \mu \mathrm{M}$ DMA; (b) $100 \mu \mathrm{M}$ bumetanide only; and (c) $100 \mu \mathrm{M}$ DMA only. One group of tissues was treated only with buffer with no inhibitors (control). After 45 minutes of incubation with/without the inhibitor(s), acetylcholine (ACh) $(100 \mu \mathrm{M})$ was added to all tissues to induce mucus secretion. After 2 hours of exposure to $\mathrm{ACh}$, the secreted mucus was collected from the cranial end of the trachea, where it pooled as a consequence of mucociliary transport.

With no inhibitor pretreatment (control), $198 \pm 89 \mu$ (mean \pm SD) of mucus was recovered from the tracheas after ACh stimulation. These mucus samples contained $600 \pm 274 \mu \mathrm{g} / \mathrm{ml}$ (mean $\pm \mathrm{SD}$ ) total mucin (Figure $5 \mathrm{~F}$ ). In this control mucus, the majority of the Muc5b population was recovered from the low-density region of the gradient (fractions 3-5) enriched for linear forms, while only a small proportion of the Muc5b was recovered from the medium-density region (fractions 6-9) enriched for the semicompact/semilinear form, and $<1 \%-3 \%$ of the Muc $5 \mathrm{~b}$ was recovered from the high-density region of the gradient (fraction 12) as the compact form (Figure 5A and D). In contrast, the mucus from the bumetanide+DMA pretreatment group had a very low total volume, $38 \pm 26 \mu 1$, with a total mucin concentration of 3,200 $\pm 2,193 \mu \mathrm{g} / \mathrm{ml}$. Rate zonal centrifugation indicated that the mucus samples from the bumetanide+DMA-pretreated tracheas had an abnormal distribution of Muc5b forms: $10 \%-25 \%$ were from the linear form region, $20 \%-30 \%$ were from the semicompact/semilinear form region, and approximately $50 \%$ were from the same high-density region as the compact form (Figure $5 \mathrm{~A}$ ). The mucus samples from the tracheas that were pretreated with only bumetanide also yielded a relatively low volume of total mucus, $99 \pm 65 \mu \mathrm{l}$, with an average total mucin concentration of $1,692 \pm 198 \mu \mathrm{g} / \mathrm{ml}$. The profile of MUC5B on the rate zonal gradient after this pretreatment was similar to the Muc5b distribution following DMA+bumetanide pretreatment (Figure 5B and E).

To investigate the role of $\mathrm{HCO}_{3}^{-}$in the mucin unpacking process, the tracheas were pretreated with DMA. This treatment produced $112 \pm 65 \mu 1$ mucus, with an average total mucin concentration of $704 \pm$ $265 \mu \mathrm{g} / \mathrm{ml}$ (Figure 5C). The density gradient profile of the Muc5b forms indicated that inhibiting $\mathrm{HCO}_{3}^{-}$ 


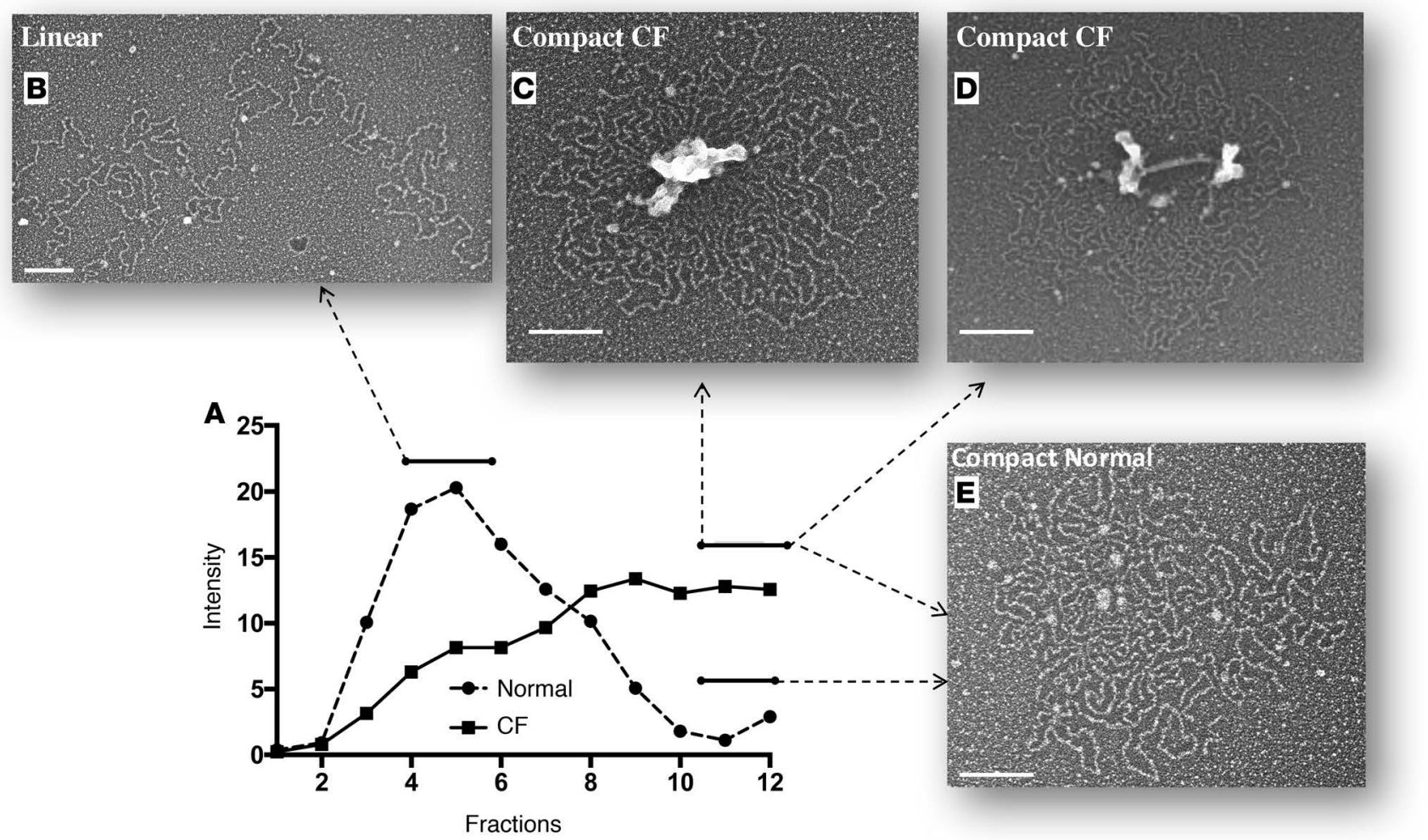

Figure 3. Transmission electron micrographs of mucin conformation reveal a defective MUC5B unpacking process in CF. Distribution of the molecular forms of salivary MUC5B from a non-CF and CF subject (A) and representative EM images from the top (B) and the bottom (C, D, E) regions. Electron microscopy showed that the compact or semiexpanding granular mucin form of MUC5B is the dominant form found at the bottom of the gradient, and it typically appears as small numbers of glycosylated mucin subunit chains connected around their $\mathrm{N}$ - and C-terminal protein nodes in spherical core $(10-30 \mathrm{~nm})$ structures $(\mathbf{E})$. In CF samples, however, an analysis of the same fractions indicated that (C and $\mathbf{D})$ the spherical protein nodes/cores are up to 10 times larger $(100-300 \mathrm{~nm})$ than $(\mathbf{E})$ their normal equivalents. Scale bars: $100 \mathrm{~nm}$.

transport altered the distribution, causing a slight decrease in the linear form and a slight increase in the compact region. However, these changes were not significant (Figure 5C). Significant decreases were observed in the linear form after bumetanide alone (Figure 5B) and DMA+bumetanide pretreatments $(P=0.01)$. Concomitantly, the proportion of the compact form increased in the samples pretreated with bumetanide alone and with DMA+bumetanide $(P=0.01)$. In addition, epithelial fluid/mucus secretion rates $(\mathrm{Jv})$ and mucin maturation were highly correlated (Figure 6). For example, in low fluid secretion rates, e.g., after treatment with bumetanide only $(1.98 \mu \mathrm{l} / \mathrm{cm} 2 / \mathrm{h})$ and DMA+ bumetanide $(0.82 \mu 1 /$ $\mathrm{cm} 2 / \mathrm{h})$, the ratios of linear mucin to compact mucin $(\mathrm{Lm} / \mathrm{Cm})$ were approximately 0.26 and 0.44 , respectively, compared with the control $(\mathrm{Jv}=6.1 \mu \mathrm{l} / \mathrm{cm} 2 / \mathrm{h}$ and $\mathrm{Lm} / \mathrm{Cm}=3.9)$. The mucin in the pig airways pretreated with DMA had a slightly increased $\mathrm{Jv}(7.8 \mu 1 / \mathrm{cm} 2 / \mathrm{h}$ ), and the $\mathrm{Lm} / \mathrm{Cm}$ was 1.17 (Figure 6).

Transmission electron microscopy analysis of the mucus samples from the treated and untreated pig tracheas indicated that the Muc5b unpacking process in CF-like airways was defective (Figure 7). The mucus from pig tracheas treated with DMA+ bumetanide or bumetanide alone indicated that the compact or semiexpanding granular mucin form of Muc5b was the dominant mucin form found at the bottom of the gradient. As in the saliva experiments (Figure 3), the compact form in nontreated samples typically had nodes measuring approximately 10-30 nm (Figure 6E). In the treated samples, however, the spherical protein nodes/cores were not as well organized and/or processed and were up to 10 times larger (100-300 nm) than normal (Figure 7, C and D).

\section{Discussion}

The transportability of the mucus layer on airway surfaces by the cilia is essential for maintaining normal lung health. This mucociliary clearance function of the airway epithelia is compromised in some diseases, notably $\mathrm{CF}$, in which the mucus accumulates in and obstructs the airways, resulting in microbiological colonization. 
The pathogenic mechanism of this mucus obstruction is incompletely understood. The results of the present study provide evidence that an abnormal fluid environment on the airway surfaces corrupts the normal unfolding process of the MUC5B mucin glycoprotein, leading to the secretion of a dense, compact form of MUC5B that likely impairs its clearance by cilia.

The biophysical properties of the airway mucus layer are strongly influenced by qualitative and quantitative changes in mucins, the dominant macromolecules in airway mucus. Our group has previously shown that mucus secretions from CF lungs contain hyperconcentrated mucins that alter the osmotic pressure dynamics between the mucus and periciliary liquid layers and play a key a role in disease pathogenesis (13). The contribution of the qualitative properties of mucins, e.g., their postsecretory unfolding processes, to disease progression is not well understood. Here, we provide evidence that qualitative changes in mucins in the CF lung might also explain the abnormal mucus properties in CF. These studies highlight the importance of postsecretory airway surface conditions for mucin unfolding and the importance of defective transepithelial $\mathrm{Cl}^{-}, \mathrm{HCO}_{3}^{-}$, and liquid secretion to the structural transformation of mucin macromolecules.

In this study, we demonstrated the postsecretory mucin unfolding and maturation abnormalities pertinent to CF airway disease and identified the dominant mechanism of defective unfolding. First, we found that mucins are released in compact granular forms and normally transform into linear forms over time. Second, we demonstrated that this process is defective in the liquid environment characteristic of the $\mathrm{CF}$ airway surface. Finally, we ascertained that, independent from lower $\mathrm{HCO}_{3}{ }^{-}$and $\mathrm{pH}$ levels, airway surface dehydration is a major determinant in this unfolding process. It is apparent that this maturation process, in which the granular compact mucins transform to the linear form, is critical for having transportable mucus in the airways. This unfolding/maturation process, however, may be unfavorable in other mucosal surfaces, e.g., the stomach and intestine, in which the mucosal surface needs a tighter barrier that does not require mucus transport over the cilia. Therefore, this ordered process is likely to be dynamically controlled by the mucosal systems according to their functional requirements.

Following exocytotic secretion in normal airways, mucin molecules rapidly expand in the surface liquid of airways, hydrating them and ultimately forming a mucus gel. This process is essential to producing a transportable mucus gel and depends on numerous factors, including mucin chain length, mucin charge state, ionic conditions, and the relative hydration state of the environment (14). There are essentially two ways in which mucins might be packaged during synthesis, and $\mathrm{Ca}^{2+}$ plays an important charge-shielding role in both cases. According to the Verdugo model (15), mucin oligomers may be packaged as individual, randomly tangled strands that would likely fold in regular patterns to form compact structures. More recent models described by Kesimer et al. (12), Thornton and colleagues (16), and Hansson and colleagues (17) provide evidence that mucins are packaged in a more organized, compact fashion that requires the oligomers to be connected physically in a networked array through their N- and C- terminal regions. Following secretion, the oligomeric strands in the Verdugo model (18-20) would simply expand via Donnan equilibrium as they hydrate and anneal into mucus, whereas in the Kesimer/Sheehan model, the mucin polymers would first need to be freed from the network by ionic exchange and likely proteolytic processing. Regardless of the mechanism involved, this unpacking process is critical for transforming highly compacted granular mucins into the linear strands that form the basis of transportable airway mucus that interacts with cilia shafts. It is important to know the route by which secreted mucins mature into mucus, as this has direct bearing on the development of therapies for $\mathrm{CF}$ patients.

In this study, we provide evidence of the postsecretory processing of MUC5B mucin after granular release under controlled conditions. In cultured, well-differentiated airway epithelial cells derived from non-CF and CF tissues, we show that MUC5B mucin originates in a highly compacted granular form that is processed into a linear form within minutes, with a complete transformation occurring within 4 hours. Although this observation is predicted by recent studies $(12,16)$, it was surprising, as earlier studies had shown that mucus swells within seconds after secretion $(20,21)$. Mucus swelling due to mucin unfolding after granular release depends on a number of factors, including charge status, hydrophobicity, polymer length, and the ionic conditions of the extracellular milieu (concentrations of $\mathrm{Na}^{+}, \mathrm{Ca}^{+2}$, $\mathrm{H}^{+}$, and $\mathrm{HCO}_{3}^{-}$, in particular) (14).

Using cell cultures derived from CF lungs and fresh saliva from CF patients, we provide evidence that this unpacking process is defective in CF mucus (Figure 1 and Figure 2). The postexocytotic transformation of the compact packaged form of MUC5B into the linear expanded form is apparently defective in agonistinduced (Figure 1B) and unstimulated basal secretions (Figure 1C). In CF, the absence of functional CFTR 
A
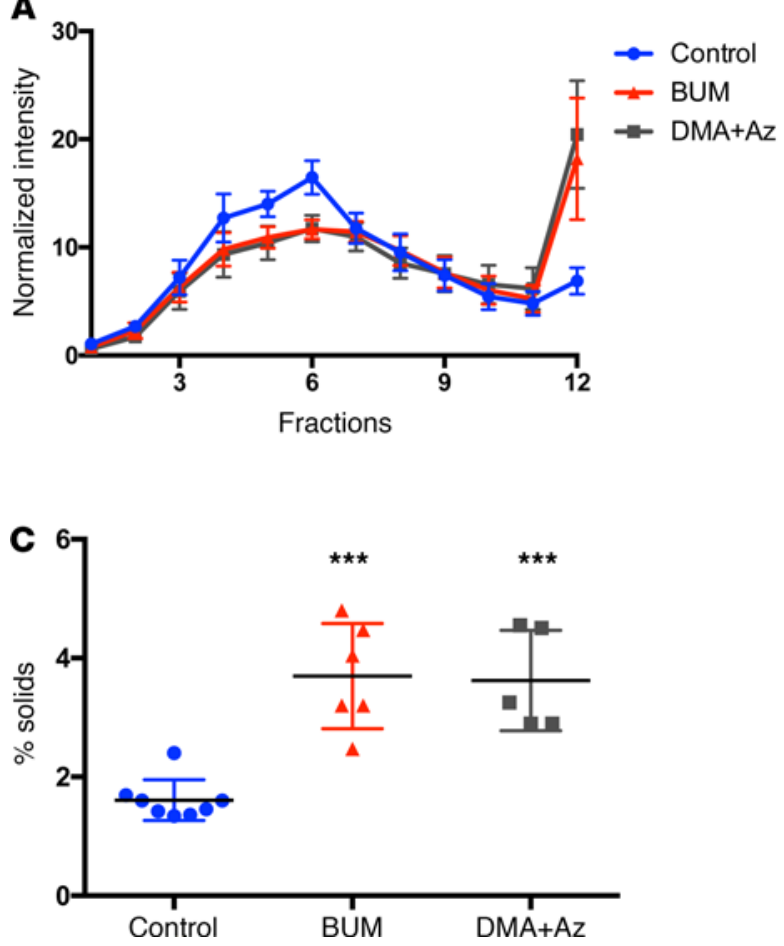
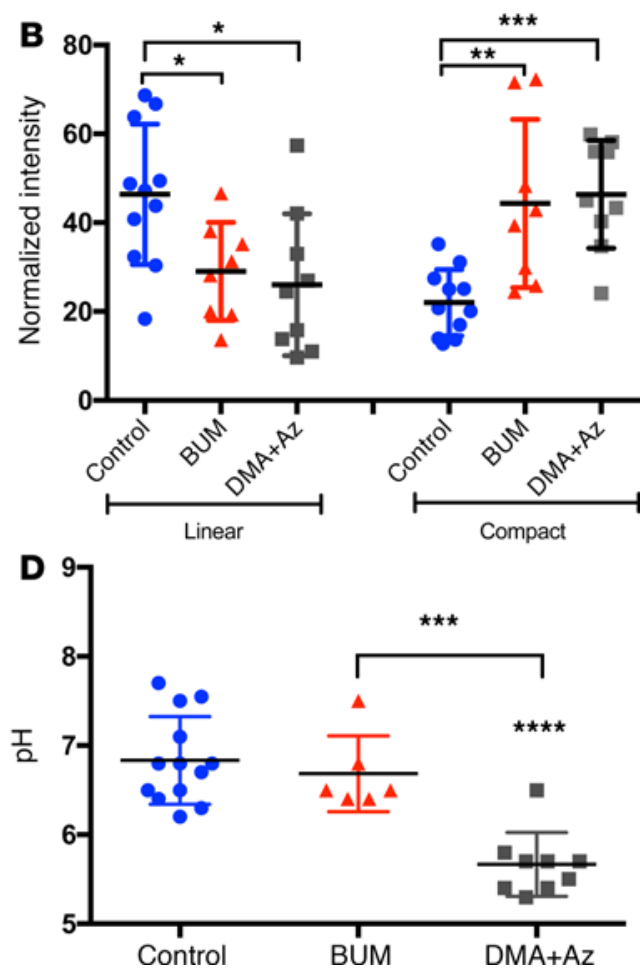

Figure 4. MUC5B distribution after treatments in culture. ATP $\gamma S$-stimulated culture secretions, after bumetanide (BUM) (10 $\mu M$ ) and dimethylamiloride+acetazolamide (DMA+Az; $100 \mu \mathrm{M})$ treatment, were subjected to a 6-8 M GuHCl rate zonal centrifugation to determine the macromolecular distribution of MUC5B. (A and B) Compared with no pretreatment control, both treatments led to significantly increased proportions of MUC5B mucin, with approximately $30 \%-40 \%$ in the high-density region ( ${ }^{*} P=0.002,{ }^{*}{ }^{*} P=0.0007$ ). The treatment samples also exhibited decreased amounts of the MUC5B form found in the light density region, compared with the control ( $\left.{ }^{*} P=0.009\right)$ (Figure 5D). (C) Both bumetanide and DMA+Az treatments increased the mucus concentration significantly ( ${ }^{* *} P=<0.005$ ), as measured by percentage solids, from 1.6\% (control) to $3.6 \%$. (D) DMA+Az treatment reduced the $\mathrm{pH}$ approximately $1 \mathrm{pH}$ unit from 6.8 (control) to $5.6{ }^{* * *} P=0.02,{ }^{* * * *} P=0.001$ ). No pH changes were observed after bumetanide treatment. Bars represent mean \pm SD. Statistical significance was determined using 1-way ANOVA.

results in a volume-depleted airway surface, reduced $\mathrm{HCO}_{3}^{-}$concentration, and a lower $\mathrm{pH}$. The loss of CFTR function has at least two consequences: (a) a dehydrated epithelial surface resulting from impaired $\mathrm{Cl}^{-}$secretion and (b) an ionically modified epithelial surface liquid caused by impaired $\mathrm{HCO}_{3}{ }^{-}$secretion. Defective $\mathrm{HCO}_{3}{ }^{-}$secretion could result in reduced $\mathrm{pH}$ on the airway surface (22) that could alter the proteolytic environment and/or impair the chelation of $\mathrm{Ca}^{+2}$ by $\mathrm{HCO}_{3}{ }^{-}(23,24)$, thereby affecting the dynamics of $\mathrm{Na}^{+} / \mathrm{Ca}^{+2}$ exchange. In both cases, the process by which mucin unfolds from a granular to a linear and more easily transportable form will be greatly affected.

The intriguing finding of an unusual, poorly processed, compact form of MUC5B observed only in CF saliva (Figure 3, C and D) also indicates that MUC5B granules are slow to unfold in the CF environment after release. Electron micrographs of the mucins secreted from pig tracheas that were pretreated with DMA+bumetanide also revealed the presence of these abnormally organized/unprocessed compact mucin forms (Figure 5, C and D). These large, irregularly shaped, poorly processed structures, which have an unprocessed protein core that contains mucins, naked protein regions, and other interacting proteins (25), render the mucins more compact and possibly more adhesive. Thus, they may bind more strongly to epithelial surfaces and the cilia and may interfere with proper ciliary beating dynamics and clearance.

It is important to understand how postsecretory mucin unpacking is compromised under CF postsecretory conditions. To create CF-like conditions in normal HTBE cell cultures, we used the well-established inhibitors DMA and bumetanide to inhibit $\mathrm{HCO}_{3}^{-}$and $\mathrm{Cl}^{-}$secretion, respectively (26). We also removed $\mathrm{HCO}_{3}{ }^{-}$from the buffer and added a carbonic anhydrase inhibitor (Az) to the DMA treatment but not to the bumetanide treatment. As summarized in Figure 4, both treatments caused a significant increase in the high-density compact form of MUC5B, suggesting that the unfolding process is greatly affected by these conditions. The bumetanide treatment caused dehydration and increased mucus concentration on the culture surfaces, as assessed by measurements of the percentage of solids, due to its inhibition of transepithelial $\mathrm{Cl}^{-}$ 


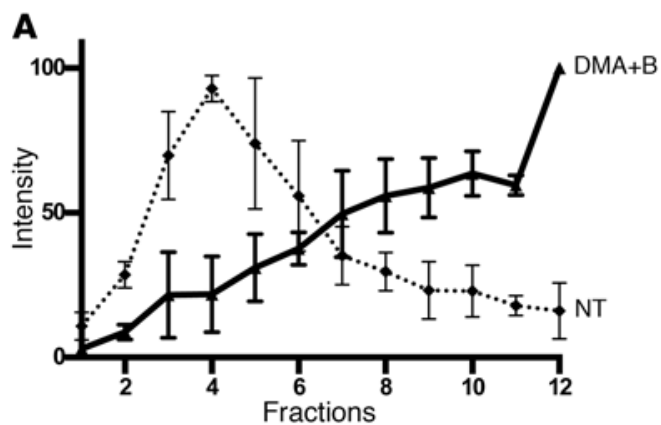

B
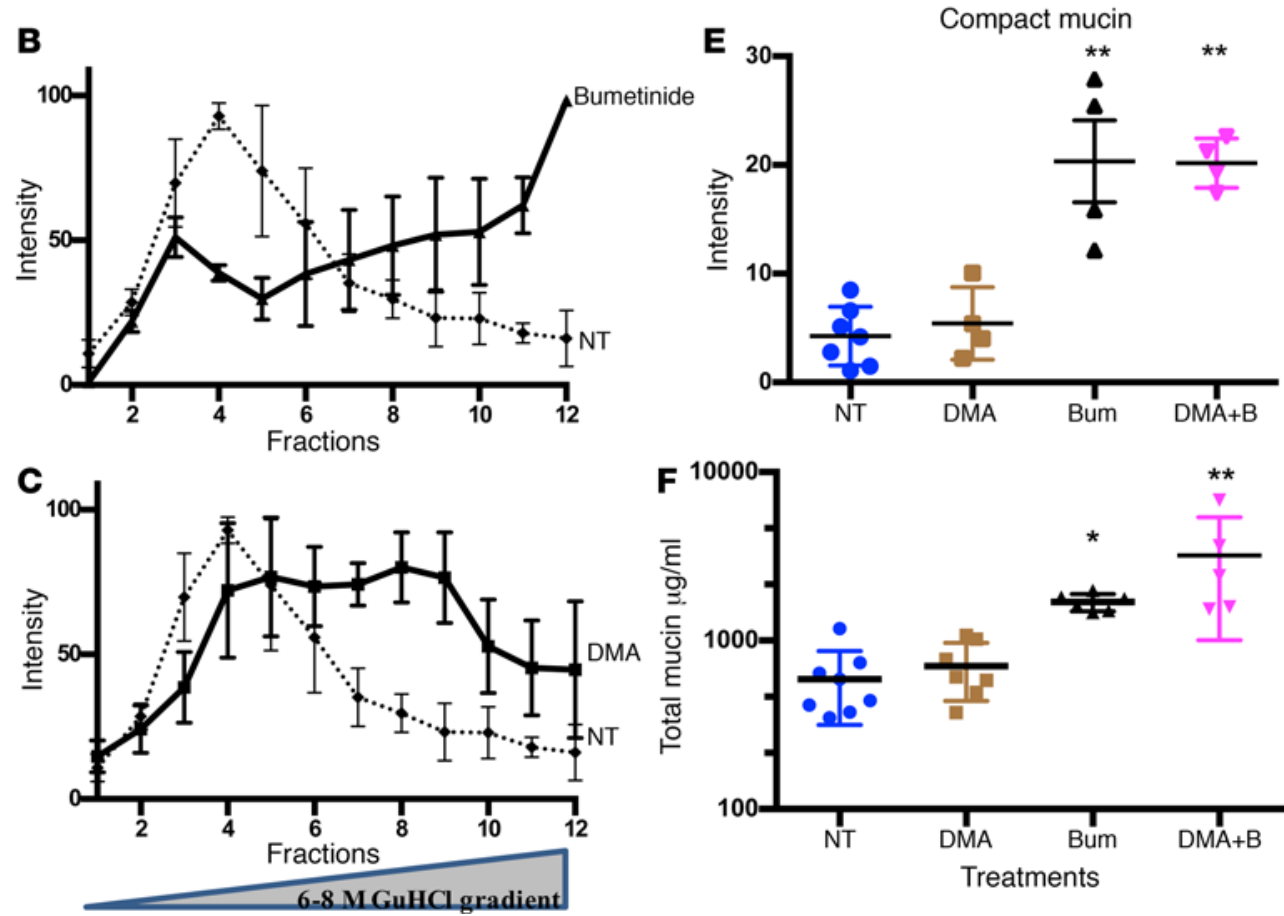

Figure 5. Distribution of mucin in CF-like pig tracheas after treatments. Four inhibitor pretreatment combinations were used: (a) $100 \mu \mathrm{M}$ bumetanide plus $100 \mu \mathrm{M}$ dimethylamiloride (DMA); (b) $100 \mu \mathrm{M}$ bumetanide; and (c) $100 \mu \mathrm{M}$ DMA as well as (d) $100 \mu \mathrm{M}$ ACh, which was added to induce mucus secretion. The control group was treated only with $100 \mu \mathrm{M}$ ACh. (A) In the control mucus, the majority of the MUC5B population was recovered on the low-density side of the gradient (top: fractions 3-5) in a linear form, while only a small proportion of the MUC5B was recovered in the medium-density region (middle: fractions 6-9) in a semicompact/semilinear form, and less than $1 \%-3 \%$ of the MUC5B was recovered in the high-density region of the gradient (bottom: fraction 12 ) in a compact form. In contrast, mucus from the bumetanide+DMA pretreatment group had an abnormal distribution of MUC5B forms: 10\%-25\% were in a linear form, 20\%-30\% were in a semicompact/semilinear form, and approximately $50 \%$ were in a compact form in the high-density region (A). (B) Mucus samples from tracheas that were pretreated with only bumetanide also yielded an abnormal Muc5b profile, as did samples pretreated with DMA+bumetanide. (C) DMA treatment slightly but not significantly changed the Muc5b profile. Significant $(P=0.005)$ decreases were observed in the linear form after $(\mathbf{B})$ bumetanide alone and $(\mathbf{A})$ DMA+bumetanide pretreatments. The proportion of the compact form significantly $(P=0.005)$ increased after bumetanide alone and DMA+bumetanide pretreatments. (D-F) Analysis of the linear and compact forms in these treatments compared with the nontreated samples is displayed as a scatter plot. Bars represent mean $\pm \operatorname{SD}\left({ }^{*} P=0.01,{ }^{* *} P=\right.$ 0.005). Statistical significance was determined using 1-way ANOVA. NT, not treated. secretion. This resulted in a substantial shift in MUC5B from the linear to the compact form. DMA+Az treatment reduced the $\mathrm{pH}$ dramatically, while increasing mucus concentration. A role for $\mathrm{HCO}_{3}^{-}$secretion in this process cannot be ruled out. A recent study suggested that both $\mathrm{pH}$ and $\mathrm{Ca}^{++}$concentration can influence ASL viscosity (27). As explained above, these factors can substantially affect the unfolding of mucin from a compact to a linear form.

To complement our cell culture studies, we also performed experiments using porcine tracheas as a model. Previous studies have demonstrated that thick airway mucus resembling the mucus observed in $\mathrm{CF}$ airways can be generated in genetically normal domestic pig tracheas in vitro by inhibiting active $\mathrm{Cl}^{-}$and $\mathrm{HCO}_{3}^{-}$secretion in the presence of $\mathrm{ACh}$, a stimulant of submucosal gland secretion $(26,28)$. This model mimics the $\mathrm{CF}$ condition by greatly reducing $\mathrm{Cl}^{-} / \mathrm{HCO}_{3}^{-}$and volume through the pharmacological inhibition of NKCC and NHE transporters in the basolateral membrane of epithelial secretory cells. In the presence of a mucus secretagogue, such as ACh, mucin secretion from the mucous cells of the submucosal glands is induced, and $\mathrm{Cl}^{-}$and $\mathrm{HCO}_{3}{ }^{-}$secretion is induced to drive fluid secretion and generate well-hydrated mucus. When bumetanide and DMA were used to inhibit NKCC and NHE, respectively, the resulting secreted mucus contained much less salt and $\mathrm{H}_{2} \mathrm{O}$, resulting in a thick, hyperconcentrated, viscous mucus with physical properties resembling those of $\mathrm{CF}$ mucus (29). It was shown that the mucociliary transport rate is dramatically reduced in these conditions (30).

An analysis of mucin forms after rate zonal centrifugation indicates that this condition has the greatest effect on mucin unpacking, i.e., it results in the highest percentage of compact forms and the lowest percentage of linear forms observed across all treatment conditions. Bumetanide-only treatment resulted in a similar trend, i.e., significant increases and decreases in the compact and linear forms, respectively. As these two conditions had normal levels of bicarbonate $(25 \mathrm{mM})$ in the buffer, we can surmise that 


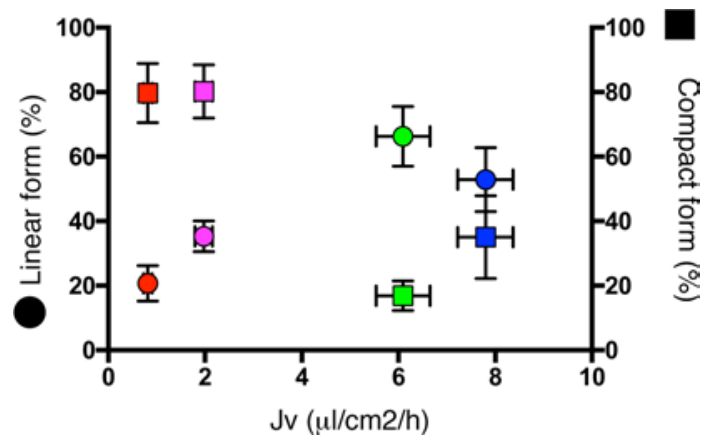

Figure 6. Correlation between the rate of liquid volume secretion and mucin maturation. Correlation between the rate of liquid volume secretion (Jv) and mucin maturation, as assessed by the percentage of linear (colored circles) and compact forms (colored squares) in control (green), dimethylamiloride (DMA) alone (blue), bumetanide alone (magenta), and DMA+bumetanide (red) treatments of pig bronchi. Horizontal and vertical bars represent \pm SD. surface dehydration is an important determinant of the unpackaging process. The greater effect of bumetanide than DMA alone in inhibiting the maturation process is logically related to the fractional effects of the two anion secretion inhibitors, as NKCC-dependent $\mathrm{Cl}^{-}$secretion drives a much greater percentage of liquid secretion in bronchial airways than $\mathrm{Na}^{+} / \mathrm{H}^{+}$exchange-dependent $\mathrm{HCO}_{3}{ }^{-}$secretion (26). This is complementary to the observations made in cell culture experiments. Figure 6 illustrates the electron microscopy analysis of the high-density region of DMA+bumetanide-treated samples, including the compact forms that typically have large, irregularly shaped, amorphous structures, which were also observed in human CF saliva.

Regardless of the underlying cause, the dominance of unfolded, compact mucins in ASL may have adverse consequences for mucus clearance. For instance, the exposed/unprocessed protein cores of the granular/compact form are more likely to strongly interact via adhesive interactions with epithelial surfaces, particularly cilia. The increased interactions with epithelial surfaces and cilia are predicted to interfere with effective mucus clearance. Additionally, mucus dominated by compact mucins may possess different biophysical properties that may also effect mucociliary clearance. Indeed, mucus released from newborn CF pig airways in response to cholinergic stimulation was strongly attached to epithelial surfaces and collapsed mucociliary transport (31), and Ballard et al. (32) showed that bumetanide+DMAtreated mucus, in which the compact mucin is dominant, as described here, was associated with a significant reduction in mucociliary transport. Further, in addition to its effect on mucin/mucus biology and biophysics, the abnormal maturation process provides information about the local environment into which mucins are released and can be used as a surrogate marker of the hydration status of the airway surfaces.

In summary, gel-forming mucins in the airways undergo a postsecretory unfolding/maturation process after granular release, and this process is defective in the CF environment due to the abnormal airway surface milieu resulting from CFTR malfunction. Our data suggest that the airway surface hydration state, which is dynamically controlled by the CTFR-related secretion of both $\mathrm{Cl}^{-}$and $\mathrm{HCO}_{3}^{-}$, appears to be a critical factor in the maturation/unfolding process. Defective maturation produces mucins in a highly cross-linked, compact form that is likely to have aberrant biophysical properties that may contribute to the defective mucociliary clearance observed in CF airways. Our ongoing studies focus on understanding the swelling and other biophysical dynamics associated with the defective maturation process to identify therapies targeted at mucus obstruction in CF.

\section{Methods}

Primary human airway epithelial cell cultures. Human tracheobronchial cells from donors were obtained in accordance with protocols approved by the IRB of the University of North Carolina at Chapel Hill, as described previously (33). Cells from non-CF and CF human bronchi were isolated and grown on plastic culture dishes in bronchial epithelial cell growth medium. The cells were passaged at $80 \%$ confluence, and first/second-passage cells were seeded onto 30- $\mathrm{mm}$ Millicell supports at $0.8-1.0$ million cells per support (Corning). At confluence, the cells were maintained under air-liquid interface (ALI) conditions in ALI culture medium, which was changed at the basolateral surface 3 times a week. HTBE cells were used for experiments 4-6 weeks after confluence, when the columnar cells were well differentiated into ciliated and goblet cells.

Mucin maturation experiments. HTBE cell cultures derived from non-CF and CF subjects were used to study the maturation of freshly secreted mucins. The cultures were carefully washed with $1 \mathrm{ml}$ PBS, as described previously (34), to remove the mucus that accumulated on the luminal surfaces of cultures during the 2-3 days between culture maintenance under ALI conditions. The accumulated mucus wash was added to an equal volume of $8 \mathrm{M} \mathrm{GuHCl}$. After a 4-hour washing protocol, the cultures were treated apically with $100 \mu \mathrm{M}$ ATP $\gamma \mathrm{S}$ (30 $\mu \mathrm{l}$ in ALI, pH 7.2 for a 30-mm culture insert, sufficient to form a thin layer over the apical surface) to stimulate mucin release (35). The stimulated apical secretions were collected in PBS $(0.1 \mathrm{ml})$ at 2, 60, and 240 minutes after ATP challenge (not sequentially — individual cultures were used for each time point) and immediately added to $8 \mathrm{M} \mathrm{GuHCl}$ to a final concentration of $4 \mathrm{M}$ to preserve the molecular forms of secreted mucins. The apical secretions were used to study the molecular 
maturation forms of freshly secreted mucins relative to the time course and the accumulated mucins by rate zonal centrifugation, as described below.

Treatments of HTBE cell cultures. To mimic the behavior of $\mathrm{CF}$ airways in which both $\mathrm{Cl}^{-}$and $\mathrm{HCO}_{3}^{-}$ secretions are impaired, the effect of anion secretion blockers on HTBE cell mucus secretion was investigated. HTBE cell cultures were washed and pretreated basolaterally for 1 hour with (a) $100 \mu \mathrm{M}$ bumetanide, an inhibitor of the basolateral $\mathrm{Na}^{+}-\mathrm{K}^{+}-2 \mathrm{Cl}^{-}$cotransporter, in ALI medium with $25 \mathrm{mM} \mathrm{HCO}_{3}{ }^{-}$and were kept in an incubator with $5 \% \mathrm{CO}_{2}$ and $95 \% \mathrm{O}_{2}$ during the entire experiment, with a set of untreated cultures as controls, or with (b) both DMA (100 $\mu \mathrm{M}$, a Na $\mathrm{Na}^{+} / \mathrm{H}^{+}$exchange inhibitor) and $\mathrm{Az}(100 \mu \mathrm{M}$, a carbonic anhydrase inhibitor) in $\mathrm{HCO}_{3}^{-}$-free, HEPES-buffered $\mathrm{ALI}$ and were kept in an $\mathrm{HCO}_{3}^{-}$-free system during the entire experiment. Control and pretreated cultures were exposed to ATP $\gamma \mathrm{S}$ in test media, as described above. Samples were collected after 4 hours. To analyze the molecular forms of mucin using rate zonal centrifugation, apical secretions were collected in PBS and immediately added to $8 \mathrm{M} \mathrm{GuHCl}$ to a final concentration of $4 \mathrm{M}$. To measure the percentage of solids and the $\mathrm{pH}$, apical secretions $(\sim 25-30 \mu \mathrm{l})$ were collected and analyzed immediately.

Rate zonal centrifugation. Samples were subjected to centrifugation on a $\mathrm{GuHCl}$ isokinetic density gradient to study the maturation of freshly secreted and accumulated mucins in normal and CF cell cultures, as described previously (36). Guanidinium chloride $(\mathrm{GuCl})$ gradients $(6-8 \mathrm{M})$ were formed in centrifuge tubes using a gradient maker (ISMATEC) connected to a Gilson Minipuls 2 peristaltic pump. Mucin samples (in $4 \mathrm{M} \mathrm{GuCl}$ ) were loaded onto the tops of the gradients and centrifuged in a Beckman Optima L-90K Ultracentrifuge (Beckman SW40 rotor) at $285,000 \times \mathrm{g}$ for 2.5 hours at $15^{\circ} \mathrm{C}$.

Fractions were assayed using slot blots probed with a monoclonal MUC5B antibody (EU-MUC5B) that recognizes the peptide sequence RNREQVGKFKMC located in the globular, Cys-rich subdomains within the PTS/mucin tandem repeat domains (37) and a polyclonal MUC5B antibody (7). The secondary antibodies used were 680 -nm goat anti-mouse and 800 -nm goat anti-rabbit antibodies (LICOR Biosciences). The signals were detected and analyzed using the Odyssey imaging system (LICOR Biosciences).

Measurements of the percentage of solids and the $p H$ of the HBE secretions. An aliquot $(20 \mu \mathrm{l})$ of HBE secretions was weighed on a microbalance (Mettler Toledo UMX2 Microbalance), dried, and weighed again. LabView software was used to record the wet and dry weights and the calculation of percentage of solids (31). A Medical Measurement System (MSS) Orion $11 \mathrm{pH}$ meter and a Greenfield single-use $\mathrm{pH}$ catheter (MSS) were used to measure the $\mathrm{pH}$ of the apical secretions collected under various treatment conditions. Treatment solutions applied to the cultures were used as a control.

Collection and initial processing of saliva. Unstimulated saliva was collected from 8 healthy volunteers and $9 \mathrm{CF}$ patients in a clinic. Saliva was collected into a specimen cup on ice. The collected saliva was initially centrifuged at $3,000 \mathrm{~g}$ for 10 minutes at $4^{\circ} \mathrm{C}$ to remove cells.

A linear sucrose gradient (10\%-30\%) in PBS with protease inhibitors (Roche Diagnostics) was created using a gradient maker and aliquoted into 12 centrifuge tubes. An aliquot from the supernatant of the saliva was layered onto the gradient. The gradients were then centrifuged for 1 hour at 210,000 $\mathrm{g}$ at $4^{\circ} \mathrm{C}$ in an SW-40 swinging bucket rotor. After centrifugation, 1-ml fractions were unloaded from the top and subjected to periodic acid-Schiff and MUC5B immunoblotting.

Collection of pig airway mucus. Domestic pigs were purchased from the Auburn University Swine Research and Education Center (Auburn, Alabama, USA). The pigs (approximately 6-12 kg) were sedated with intramuscular telazol $(26 \mathrm{mg} / \mathrm{kg})$ and ketamine $(18 \mathrm{mg} / \mathrm{kg})$ and euthanized with an overdose of pentobarbital administered through an ear vein. The tracheas were sectioned immediately below the larynx and above the first bronchial branch and removed. The tracheas were then placed in $\mathrm{HCO}_{3}^{-}$-buffered $\mathrm{Krebs}$ solution at room temperature and gradually warmed to $37^{\circ} \mathrm{C}$ over approximately 45 minutes. The Krebs solution was constantly bubbled with $95 \% \mathrm{O}_{2}$ and $5 \% \mathrm{CO}_{2}$ throughout the experiment.

The tracheas were then removed from the bath solution, and the trachealis muscle was resected along its length. Each end of the trachea was carefully tied with a heavy suture onto Lucite cannulas that were lightly coated with silicone grease. The cannulas were supported in a rack that held the trachea horizontally in space so that the open slot was oriented upward, allowing access to the ventral mucosal surface (for a depiction of this apparatus, see the online supplement for Ballard et al., ref. 30). The rack holding the trachea was placed in a weighted polycarbonate box that was partially submerged in a $37^{\circ} \mathrm{C}$ heated water bath. Warm $\left(37^{\circ} \mathrm{C}\right)$ Krebs solution was added to the box to bathe the outer surface of the trachea, but no bath solution was allowed to spill into the tracheal lumen. A tempered glass lid was placed on the box to 


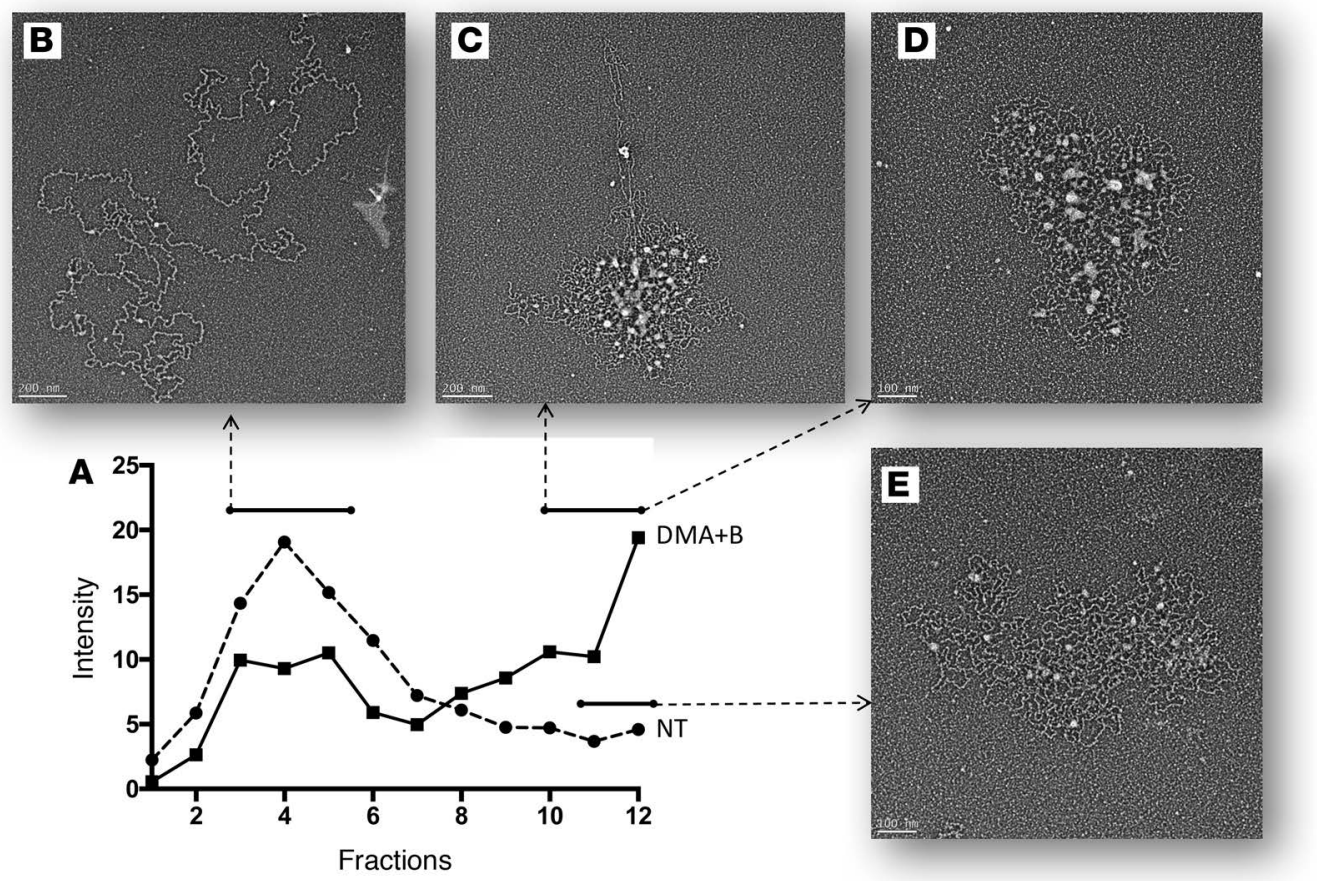

Figure 7. Transmission electron microscopy analysis of mucin conformation reveals a defective mucin unpacking process in CF-like pig tracheal mucus. A typical distribution of the molecular forms of pig tracheal Muc $5 \mathrm{~b}$ in control and CF-like pig trachea (A) and representative EM images from the top (B) and the bottom (C, D and E) regions. (E) As in the saliva experiments (Figure 3), the compact form in nontreated samples typically possessed 10 - to 30 -nm nodes. (C and D) In the treated samples, however, spherical protein nodes/cores were not as well organized and/or processed and were up to 10 times larger $(100-300 \mathrm{~nm})$ than the normal equivalents. Scale bars: $100 \mathrm{~nm}$.

maintain a warm, humidified environment inside the chamber; it was heated to prevent condensation on the inner surface of the lid. The Krebs bath was constantly bubbled with $5 \% \mathrm{CO}_{2}$ in $\mathrm{O}_{2}$ gas to keep the $\mathrm{pH}$ of the solution close to 7.4 .

The tracheas were exposed to the following pretreatments: $100 \mu \mathrm{M}$ DMA; $100 \mu \mathrm{M}$ bumetanide; a combination DMA+bumetanide solution; and no inhibitor pretreatment, which served as the control. The tracheas were exposed to the pretreatment condition for 45 minutes. Then, $100 \mu \mathrm{M}$ ACh was added to the bath to induce mucus secretion. After 2 hours, the secreted mucus was collected with pipettes, transferred to Eppendorf tubes, sealed with parafilm, and refrigerated at $4^{\circ} \mathrm{C}$.

The liquid volume secretion rates $\left(\mathrm{J}_{\mathrm{v}}={ }_{\mu l . c m}{ }^{2} \cdot \mathrm{h}^{-1}\right)$ in the control and treatment groups were calculated as described in detail elsewhere (38).

Electron microscopic analysis. For the electron microscope analysis, the concentration of the mucin pool was adjusted to approximately $10 \mu \mathrm{g} / \mathrm{ml}$. The samples were fixed with $0.6 \%$ glutaraldehyde for 4 minutes at $20^{\circ} \mathrm{C}$ and mounted on a thin glow-charged carbon-coated grid for 5 minutes. The samples were then washed in water-ethanol, as described elsewhere (39). The samples were shadow cast with tungsten and observed with a Tecnai 12 electron microscope (FEI) at $40 \mathrm{kV}$. Images were taken at $\times 30,000$ or $\times 45,000$ magnification on Kodak SO163 films and scanned with an Imacon 848 film scanner; the contrast was optimized, and panels were arranged using Adobe Photoshop software.

Statistics. Statistical analyses were performed using 1-way ANOVA and standardized 1tailed $t$ tests where applicable. A $P$ value of less than 0.05 was considered significant.

Study approval. Human tracheobronchial cells from donors and the non-CF and CF saliva were obtained in accordance with protocols approved by the IRB of the University of North Carolina at Chapel Hill. For the animal studies, all procedures involving swine were approved by the University of South Alabama Institutional Animal Care and Use Committee and complied with the United States Public Health Service Policy on Humane Care and Use of Laboratory Animals. 


\section{Author contributions}

MK, STB, CWD, and LHA designed the research studies and wrote the manuscript. LHA, JRE, TW, AAF, and $\mathrm{KN}$ performed the experiments. JDG and AMM designed and performed the electron microscopy experiments. RDC collected saliva from the CF subjects. LHA, JRE, AMM, CWD, STB, and MK analyzed the data.

\section{Acknowledgments}

This study was supported by grants from the Cystic Fibrosis Foundation (KESIME10I0), the Cystic Fibrosis Foundation Therapeutics (Mucociliary Clearance Consortium) (KESIME14XX0 and BALLARD07XX0), and the National Institutes of Health (R01HL103940 to MK, PI).

Address correspondence to: Mehmet Kesimer, Marsico Lung Institute, UNC CF Center, 7111 Marsico Hall, Chapel Hill, North Carolina 27599-7248, USA. Phone: 919.843.2577; E-mail: mehmet_kesimer@med.unc.edu.

1. Boucher RC. Cystic fibrosis: a disease of vulnerability to airway surface dehydration. Trends Mol Med. 2007;13(6):231-240.

2. Kreda SM, Gentzsch M. Imaging CFTR protein localization in cultured cells and tissues. Methods Mol Biol. 2011;742:15-33.

3. Engelhardt JF, Zepeda M, Cohn JA, Yankaskas JR, Wilson JM. Expression of the cystic fibrosis gene in adult human lung. J Clin Invest. 1994;93(2):737-749.

4. LeSimple P, et al. Cystic fibrosis transmembrane conductance regulator is expressed in mucin granules from Calu-3 and primary human airway epithelial cells. Am J Respir Cell Mol Biol. 2013;49(4):511-516.

5. Kreda SM, Davis CW, Rose MC. CFTR, mucins, and mucus obstruction in cystic fibrosis. Cold Spring Harb Perspect Med. 2012;2(9):a009589.

6. Boucher RC. Evidence for airway surface dehydration as the initiating event in CF airway disease. J Intern Med. 2007;261(1):5-16.

7. Kesimer M, Ehre C, Burns KA, Davis CW, Sheehan JK, Pickles RJ. Molecular organization of the mucins and glycocalyx underlying mucus transport over mucosal surfaces of the airways. Mucosal Immunol. 2013;6(2):379-392.

8. Groneberg DA, et al. Expression of MUC5AC and MUC5B mucins in normal and cystic fibrosis lung. Respir Med. 2002;96(2):81-86

9. Hovenberg HW, Davies JR, Carlstedt I. Different mucins are produced by the surface epithelium and the submucosa in human trachea: identification of MUC5AC as a major mucin from the goblet cells. Biochem J. 1996;318 (Pt 1):319-324.

10. Kesimer M, et al. Tracheobronchial air-liquid interface cell culture: a model for innate mucosal defense of the upper airways? Am J Physiol Lung Cell Mol Physiol. 2009;296(1):L92-L100.

11. Roy MG, et al. Muc5b is required for airway defence. Nature. 2014;505(7483):412-416.

12. Kesimer M, Makhov AM, Griffith JD, Verdugo P, Sheehan JK. Unpacking a gel-forming mucin: a view of MUC5B organization after granular release. Am J Physiol Lung Cell Mol Physiol. 2010;298(1):L15-L22.

13. Henderson AG, et al. Cystic fibrosis airway secretions exhibit mucin hyperconcentration and increased osmotic pressure. $J$ Clin Invest. 2014;124(7):3047-3060.

14. Verdugo P. Supramolecular dynamics of mucus. Cold Spring Harb Perspect Med. 2012;2(11):a009597.

15. Verdugo P, Deyrup-Olsen I, Aitken M, Villalon M, Johnson D. Molecular mechanism of mucin secretion: I. The role of intragranular charge shielding. J Dent Res. 1987;66(2):506-508.

16. Ridley C, et al. Assembly of the respiratory mucin MUC5B: a new model for a gel-forming mucin. J Biol Chem. 2014;289(23):16409-16420.

17. Ambort D, et al. Calcium and $\mathrm{pH}$-dependent packing and release of the gel-forming MUC2 mucin. Proc Natl Acad Sci USA. 2012;109(15):5645-5650.

18. Aitken ML, Verdugo P. Donnan mechanism of mucin release and conditioning in goblet cells: the role of polyions. Symp Soc Exp Biol. 1989;43:73-80.

19. Tam PY, Verdugo P. Control of mucus hydration as a Donnan equilibrium process. Nature. 1981;292(5821):340-342.

20. Tam PY, Verdugo P. Mucus hydration: a Donnan equilibrium controlled process. Adv Exp Med Biol. 1982;144:101-103.

21. Verdugo P. Mucin exocytosis. Am Rev Respir Dis. 1991;144(3 Pt 2):S33-S37.

22. Coakley RD, et al. Abnormal surface liquid $\mathrm{pH}$ regulation by cultured cystic fibrosis bronchial epithelium. Proc Natl Acad Sci USA. 2003;100(26):16083-16088.

23. Chen EY, Yang N, Quinton PM, Chin WC. A new role for bicarbonate in mucus formation. Am J Physiol Lung Cell Mol Physiol. 2010;299(4):L542-L549.

24. Quinton PM. Role of epithelial $\mathrm{HCO}^{-}$transport in mucin secretion: lessons from cystic fibrosis. Am J Physiol, Cell Physiol. 2010;299(6):C1222-C1233.

25. Radicioni G, et al. The innate immune properties of airway mucosal surfaces are regulated by dynamic interactions between mucins and interacting proteins: the mucin interactome. Mucosal Immunol. 2016;9(6):1442-1454.

26. Trout L, King M, Feng W, Inglis SK, Ballard ST. Inhibition of airway liquid secretion and its effect on the physical properties of airway mucus. Am J Physiol. 1998;274(2 Pt 1):L258-L263.

27. Tang XX, et al. Acidic pH increases airway surface liquid viscosity in cystic fibrosis. J Clin Invest. 2016;126(3):879-891.

28. Cooper JL, Quinton PM, Ballard ST. Mucociliary transport in porcine trachea: differential effects of inhibiting chloride and bicarbonate secretion. Am J Physiol Lung Cell Mol Physiol. 2013;304(3):L184-L190.

29. Ballard ST, Inglis SK. Liquid secretion properties of airway submucosal glands. J Physiol (Lond). 2004;556(Pt 1):1-10. 
30. Ballard ST, Parker JC, Hamm CR. Restoration of mucociliary transport in the fluid-depleted trachea by surface-active instillates. Am J Respir Cell Mol Biol. 2006;34(4):500-504

31. Button B, et al. A periciliary brush promotes the lung health by separating the mucus layer from airway epithelia. Science. 2012;337(6097):937-941.

32. Ballard ST, Trout L, Mehta A, Inglis SK. Liquid secretion inhibitors reduce mucociliary transport in glandular airways. Am J Physiol Lung Cell Mol Physiol. 2002;283(2):L329-L335.

33. Fulcher ML, Gabriel S, Burns KA, Yankaskas JR, Randell SH. Well-differentiated human airway epithelial cell cultures. Methods Mol Med. 2005;107:183-206.

34. Abdullah LH, Wolber C, Kesimer M, Sheehan JK, Davis CW. Studying mucin secretion from human bronchial epithelial cell primary cultures. Methods Mol Biol. 2012;842:259-277.

35. Abdullah LH, et al. P2u purinoceptor regulation of mucin secretion in SPOC1 cells, a goblet cell line from the airways. Biochem J. 1996;316 (Pt 3):943-951.

36. Sheehan JK, Carlstedt I. Size heterogeneity of human cervical mucus glycoproteins. Studies performed with rate-zonal centrifugation and laser light-scattering. Biochem J. 1987;245(3):757-762.

37. Rousseau K, Wickstrom C, Whitehouse DB, Carlstedt I, Swallow DM. New monoclonal antibodies to non-glycosylated domains of the secreted mucins MUC5B and MUC7. Hybrid Hybridomics. 2003;22(5):293-299.

38. Martens CJ, Inglis SK, Valentine VG, Garrison J, Conner GE, Ballard ST. Mucous solids and liquid secretion by airways: studies with normal pig, cystic fibrosis human, and non-cystic fibrosis human bronchi. Am J Physiol Lung Cell Mol Physiol. 2011;301(2):L236-L246

39. Griffith JD, Christiansen G. Electron microscope visualization of chromatin and other DNA-protein complexes. Annu Rev Biophys Bioeng. 1978;7:19-35. 